\title{
A Physiological Exploration of Visual Social Media Marketing: An Abstract
}

\author{
Chinintorn Nakhata and Alexa K. Fox
}

\begin{abstract}
As visual social media marketing (VSMM) (i.e., photos and videos that are informal in appearance and are used by companies for promotional purposes) continues to increase in popularity, it is imperative for marketers to understand the elements of such marketing efforts that catch consumers' attention. As such, the present research (1) studies the effect of time pressure on consumers' attention in real time as they consume VSMM content and (2) delves into finer nuances of the effectiveness of VSMM content by examining the use of linguistic (i.e., figurative language) and pictorial (i.e., drawings) elements in VSMM content on consumers' attention. Results across three studies using eye-tracking technology and scenariobased experiments indicate that time pressure elicits greater attention to the VSMM caption than no time pressure. Furthermore, the use of figurative language (i.e., antithesis) positively influences consumers' attention to the caption in companygenerated VSMM. Finally, the presence of pictorial information in VSMM positively moderates the relationship between the use of figurative language (i.e., alliteration) and consumers' attention to the caption in user-generated VSMM.
\end{abstract}

\section{Nakhata $(\square)$}

Penn State Harrisburg, Middletown, PA, USA

e-mail: czn43@psu.edu

\author{
A. K. Fox \\ University of Akron, Akron, OH, USA \\ e-mail: aks33@uakron.edu
}

